\title{
ANÁLISIS DE LOS PROYECTOS AMBIENTALES ESCOLARES (PRAE) EN VALLEDUPAR, COLOMBIA, SEGÚN LA TEORÍA DE RACIONALIDAD AMBIENTAL DE ENRIQUE LEFF
}

\author{
Andrés Alfonso Figueroa Ramírez ${ }^{1}$ \\ Adriana María Imperador ${ }^{2}$
}

Resumen: El presente artículo es un escrito cítrico, fruto del análisis practicado a los Proyectos Ambientales Escolares (PRAE) de instituciones educativas públicas del municipio de Valledupar, Colombia. Con el objetivo de entablar una discusión sobre el tipo racionalidad embebido en los documentos oficiales y, por consiguiente, en los programas de Educación Ambiental desarrollados en las instituciones del municipio, teniendo en cuenta la teoría de Racionalidad Ambiental descrita por Enrique Leff. La teoría de Leff tiene como base la crítica a la racionalidad económica instrumental imperante y propone nuevos caminos epistemológicos que incitan a pensar en escenarios alternativos para alcanzar el desarrollo sostenible

Palabras clave: Proyectos Ambientales Escolares; Racionalidad Ambiental; Análisis Documental; Educación Ambiental en Colombia.

Abstract: This article is critical writing carried out on School Environmental Projects (PRAE, for its initials in Spanish) of public educational institutions in the municipality of Valledupar, Colombia, to discuss the kind of embedded rationality in official documents and therefore in the environmental education program developed in the educational institutions of the municipality considering the environmental rationality theory described by Enrique Leff, noted for his criticism to the prevailing economic instrumental rationality and for proposing new epistemological paths that lead to thinking about different approaches to reach the sustainable development.

Keywords: School Environmental Project; Environmental Rationality; Documentary Analysis; Environmental Education in Colombia.

1Universidade Federal de Alfenas. E-mail: andres.ramirez@sou.unifal-mg.edu.br,

Link para o Lattes: http://lattes.cnpq.br/2467600239886717

2Universidade Federal de Alfenas. E-mail: adriana.imperador@unifal-mg.edu.br.

Link para o Lattes: http://lattes.cnpq.br/6511962778909776 


\section{Introducción}

No es un secreto que afrontamos una crisis ambiental ya avisada de alguna forma por Rachel Carson en 1962 en su texto "Primavera Silenciosa" (CARSON, 1969). Una crisis agudizada por las revoluciones industriales en conjunto con un modelo económico de crecimiento sin límites, pero, sobre todo, agudizada por la racionalidad económica instrumental imperante, el logocentrismo y la incapacidad del ser humano de visualizar y comprender su relación de interdependencia con la naturaleza.

Una crisis ambiental, pero también cultural, social y económica, denominada por diferentes autores como una "crisis civilizatoria" (GALANO et al., 2002; LEFF, 2004; DE SOUZA SANTOS, 2006), causada por la cosificación y superexplotación de la naturaleza; y mediada por una relación mercantilista entre el hombre y los demás componentes bióticos y abióticos del planeta, y respaldada por un modelo hegemónico de racionalidad económica que comparte Colombia como país "subdesarrollado" en búsqueda del crecimiento económico a costa de la degradación de sus ecosistemas (RODRíGUEZ, 2009, p.19), además guiado por el eurocentrismo y orquestado por la dominación del saber (ESCHENHAGEN, 2007, p.40).

Sin embargo, fue en los países desarrollados, en la década de los setenta, donde la comunidad internacional comenzó a reflexionar sobre los límites del crecimiento y su relación con la degradación del ambiente. Como ejemplo está el documento publicado por el Club De Roma en 1972, titulado "Los límites del crecimiento" (MEADOWS et al., 1972 apud IRANZO, 2005).

Estas reflexiones tuvieron su punto de inflexión en 1972, en Estocolmo, Suecia, debido al desarrollo de la renombrada conferencia de las Naciones Unidas sobre el Medio Humano, donde se firmó un documento entre las partes con varios compromisos, entre los cuales estaba la educación en cuestiones ambientales para todas las personas (ONU, 1973, p.5), marcando el inicio de la Educación Ambiental como un pilar fundamental para la transformación social e institucional, en procura de enfoques alternativos al desarrollo (GONZÁLEZ, 2017, p.4).

El estado colombiano, en razón a los compromisos adquiridos en las conferencias internacionales, desarrolló diferentes iniciativas para la implementación de la Educación Ambiental en el territorio. Una de esas iniciativas fue implantación de Proyectos Ambientales Escolares (PRAE), los cuales debían ser incluidos en el Proyecto Pedagógico Institucional (PEI) en las escuelas oficiales y no oficiales por obligatoriedad desde enero de 1995 (MEN, 1994, p.1), convirtiéndose en la única herramienta de Educación Ambiental en el sector formal de la educación desde dicha fecha hasta la actualidad.

En ese orden de ideas, se hace imperativo reflexionar sobre la capacidad de los PRAE para formar sujetos críticos y comprometidos con su relación de interdependencia con la naturaleza en el territorio colombiano. Consecuentemente con lo anterior, se planteó la realización de este artículo en 
pro de develar el concepto de racionalidad que poseen los PRAE en el municipio de Valledupar, Colombia. Para ello, se tomó como referencia la teoría de Racionalidad Ambiental de Enrique Leff, descrita principalmente en su libro Racionalidad Ambiental: la reapropiación social de la naturaleza (2004), considerada como una alternativa analítica para una nueva relación humanomundo (BUENO; CERVEIRA, 2016, p.171).

La investigación se desarrolló en el municipio de Valledupar, que figura como capital del departamento del Cesar, ubicado al norte de Colombia. Según cifras de la Secretaría de Educación Municipal (SEM), para el 2020 el municipio contaba con una población estudiantil de 85.510 estudiantes y 2632 docentes, en sus 41 instituciones oficiales (SEM, 2020), distribuidas en el casco urbano y la zona rural con 26 y 15 establecimientos, respectivamente.

Además de tener relevancia como ciudad capital del departamento del Cesar, existen otros aspectos que acentúan el protagonismo regional de Valledupar en términos de gestión ambiental. En el territorio municipal existen áreas consignadas en el Registro Nacional de Áreas Protegidas (RUNAP), tales como: El Parque Nacional Natural de la Sierra Nevada de Santa Marta, el Parque Natural Regional los Besotes, la Reserva Forestal Regional los Ceibotes, y la Reserva Natural de la Sociedad Civil predio Paraver. Estos dos últimos corresponden a remanentes del Bosque Seco Tropical (BST), ecosistema altamente amenazado (PIZANO; GARCIA, 2014, p.9), relevante en la conservación de las especies, muchas de ellas endémicas de los ecosistemas en esas zonas.

Por otra parte, en el municipio, un buen porcentaje de la población no tiene acceso a las instituciones educativas, lo que dificulta mejorar la Educación Ambiental desde el ámbito formal. Esto se debe al aumento de la pobreza monetaria experimentado en los últimos años en el municipio. Aumento que para el año 2017 ascendía al 33.4\%, en contraste con el resto del país que mostraba una tendencia descendente; y que se relaciona con un crecimiento poblacional descontrolado debido al alto número de desplazados por el conflicto armado interno, estimado en 143.604 personas en el periodo 1996-2018 (OTERO-COTES; HERRERA; MONROY, 2019, p.34), situación que se traduce en el aumento de familias que no poseen una vivienda digna, recursos limitados y baja escolarización, lo que disminuye el contacto de los niños y adolescentes del municipio con la educación ambiental, pues al no asistir a la escuela no tienen la oportunidad de hacer parte de los PRAE diseñados específicamente para alcanzar esa parcela de la población.

En aras de alcanzar nuestro objetivo, se analizaron los documentos oficiales de 12 instituciones educativas, las cuales fueron las únicas que respondieron a nuestra petición, mediante la técnica de Análisis Documental descrita por Antonio Gil (2002). Este análisis se realizó con la finalidad de ahondar en los mensajes latentes del texto, bajo una lectura crítica realizada por los autores, para luego enfrentar los textos en una lectura cruzada con la teoría de Enrique Leff (2004) creando categorías de análisis, para 
posteriormente realizar inferencias, dando como resultado una investigación de corte cualitativo-descriptivo, organizada en las tres siguientes sesiones.

La primera sesión es una revisión histórica sobre la implementación de Ios PRAE en Colombia, necesaria para dar contexto a las demás sesiones. La segunda sesión habla sobre el concepto de racionalidad integrado en los PRAE del municipio de Valledupar. Y por último, la tercera sesión, que se enfoca en el potencial del diálogo de saberes para entablar conversaciones en las escuelas que incentiven la conciencia y responsabilidad ambiental desde las cosmovisiones locales.

\section{Recorrido por los Proyectos Ambientales Escolares en Colombia}

Los PRAE son el resultado de una de las dos grandes reformas a la política pública de gestión ambiental en Colombia, descritas por el exministro de ambiente Manuel Rodríguez (2009), como intentos de hacer más verde al estado colombiano. Así, la historia de los PRAE como estrategia pedagógica de Educación Ambiental para el sector formal es fruto de la segunda gran reforma, en 1994 con la expedición de la ley 115 o ley general de Educación (COLOMBIA, 1994a), reglamentada por el Decreto n¹743 (MEN, 1994), donde se fijaron los criterios para su promoción en el sector formal e informal, y consecutivamente, la implementación desde enero del próximo año.

Sin embargo, es válido comentar que antes de la formalización de los PRAE, existieron otras iniciativas que contextualizan la formulación de los mismo. Iniciativas que demuestran el devenir del accionar gubernamental colombiano para con la educación ambiental, generalmente estimulado por los tratados y conferencias internacionales.

En ese orden de ideas, la primera acción gubernamental volcada a la promoción de la Educación Ambiental en Colombia data de 1974, dos años después de la Conferencia de Naciones Unidas sobre el Medio Humano realizada en Estocolmo, y corresponde a la formulación del Código Nacional de Recursos Naturales Renovables y de Protección al Medio Ambiente que estipulaba en su título II, de la parte III:

Promover la realización de jornadas ambientales con participación de la comunidad, y de campañas de educación popular, en los medios urbanos y rurales para lograr la comprensión de los problemas del ambiente, dentro del ámbito en el cual se presentan (COLOMBIA, 1974, p.5).

Cuatro años después, en 1978 se expide el Decreto $n^{\circ} 1337$ (COLOMBIA, 1978) por el cual se reglamenta la implementación de la educación ecológica y la preservación ambiental en el sector educativo en Colombia. Aunque en palabras de Torres (1998, p.24), las anteriores iniciativas

Revista brasileira educação ambiental 
presentaban limitaciones por cuanto a su perspectiva fundamentalmente conservacionista propia de la época.

En 1991, con la creación de la nueva constitución política, tildada de ambientalista por contener más de cincuenta artículos que hacen referencia al medio ambiente (RODRíGUEZ et al., 2008, p.1), se crea el marco normativo general para la Educación Ambiental en Colombia, descrito en los artículos 67 y 79 que estipulan como deber del estado colombiano: (1) usar la educación como herramienta que ayude a formar al ciudadano para la protección del ambiente, (2) garantizar el derecho a gozar de un ambiente sano y proteger la diversidad e integridad del ambiente, respectivamente (COLOMBIA, 1991).

Posteriormente, en 1994, dos años después de la conferencia de las Naciones Unidas sobre el Desarrollo Sostenible en Río de Janeiro, se expide la ley 115 o ley general de la educación que, en su artículo n²3, establece la Educación Ambiental como un área obligatoria y fundamental necesaria para ofrecer en el currículo como parte del PEI, además decreta que:

Uno de los fines de la educación es la tendiente adquisición de una cultura ecológica basada en la adquisición de una conciencia para la conservación, protección y mejoramiento del medio ambiente, de la calidad de vida y del uso racional de los recursos naturales, entre otros (COLOMBIA, 1994).

Por lo tanto, desde el mes de enero de 1995 hasta la actualidad, todas las instituciones de carácter oficial y no oficial en los niveles de preescolar, básica y media, deben desarrollar dentro del marco pedagógico institucional, Proyectos Ambientales Escolares basados en diagnósticos ambientales, locales, regionales y/o nacionales (MEN, 1994, p.2); teniendo en cuenta la interculturalidad, formación en valores, regionalización, interdisciplinariedad, participación y formación para la democracia, y la gestión y resolución de problemas, como principios rectores de la educación ambiental.

Finalmente, en el 2002 se expide la Política Nacional de Educación Ambiental (PNEA), proporcionando un marco conceptual y metodológico básico que orienta las acciones que en materia educativo-ambiental que se adelanten en el país, tanto a nivel de educación formal como no formal e informal (COLOMBIA, 2002), buscando el fortalecimiento de los procesos en todos los rescoldos de la sociedad, con la finalidad de formar personas críticas a favor de las relaciones sociedad-naturaleza en el marco del desarrollo sostenible.

En la PNEA se encuentra la definición actual de Educación Ambiental que rige en el territorio colombiano, como:

El proceso que le permite al individuo comprender las relaciones de interdependencia con su entorno, a partir del conocimiento reflexivo y crítico de su realidad biofísica, social, política, económica y cultural (COLOMBIA, 2002, p.18). 
Concepto que deben tener en cuenta los dinamizadores ambientales de las escuelas o cualquier individuo que desarrolle actividades volcadas a la Educación Ambiental.

Aunque la experiencia en la implementación de los PRAE, incluidos en la PNEA en 2002, a la fecha, 2021, lleva más de dos décadas en el territorio colombiano, existen diferentes cuestionamientos a su eficiencia. Según el estudio de Huérfano (2018), es evidente que en la teoría se presenta una visión de interdependencia entre naturaleza-sociedad, sin embargo, en la realidad los dinamizadores ambientales de las instituciones muestran una inclinación meramente ecológica de la naturaleza y conservación.

Sumado a lo anterior, los PRAE no son abordados como verdaderos proyectos educativos, sino como jornadas por la defensa de algún componente del medio: atmósfera, agua, paisaje, fauna, flora, entre otros, (HERRERA et al., 2006, p.120), convirtiéndose en actividades atomizadas desprovistas de capacidad para formar sujetos capaces de asumir una posición crítica en relación con el deterioro ambiental.

Sarmiento (2018, p.140) anota que, en términos de regulación e institucionalidad la PNEA ha enfrentado serios obstáculos para su implementación efectiva, ya que en el país la idea de desarrollo se ha confundido con un crecimiento económico promovido por la sucesión de gobiernos, pues, en muchos sectores los principios de protección ambiental aún se consideran una restricción al desarrollo económico y social (LEFF et al., 2002, p.17), visión que hace caso a las pretensiones del mercado y a la racionalidad económica.

Además de los obstáculos mencionados, existen otros, como: el aislamiento de los campos disciplinares en las escuelas, el concepto del saber científico cómo verdad absoluta, el docente como el poseedor de esa verdad, y la organización vertical del sistema educativo (BUSTAMANTE; CRUZ; RIVERA, 2017, p.221); inconvenientes que deben sortear los dinamizadores ambientales, ya que minan su quehacer, pues inevitablemente hacen parte su realidad. Una realidad legitimada por las relaciones de poder sobre el saber, proveniente desde las grandes esferas de la gestión educativo-ambiental en el país.

\section{Racionalidad Ambiental y Proyectos Ambientales Escolares}

La visión de la PNEA en Colombia es "formar sujetos capaces de intervenir de manera consciente, crítica y a favor en la relación sociedadnaturaleza en el marco del desarrollo sostenible" (COLOMBIA, 2002, p.18), donde los PRAE tienen un papel fundamental por ser la única herramienta para cumplir esa misión en el sector formal de la educación. Por lo tanto, es imperativo dilucidar bajo qué tipo de racionalidad productiva es impartida la educación para llegar al tan anhelado desarrollo sostenible, tomando como principio rector la idea de Leff sobre una solución diferenciada a la problemática

Revista brasileira educação ambiental 
ambiental. El autor explica que la solución está en "la construcción de un nuevo paradigma productivo fundado en la productividad ecológica, los valores culturales, los significados subjetivos y la creatividad humana" (LEFF, 2004, p.191).

Vale la pena detenernos a reflexionar sobre el impacto de los PRAE en la sociedad colombiana, caso específico, la sociedad Valduparense. Como ya se expresó, los PRAE son las únicas iniciativas de Educación Ambiental en el sector formal de la educación Colombiana, con la capacidad y responsabilidad de crear un nuevos Ethos del desarrollo basado en los principios culturales, alejado de la racionalidad económica globalizante, ya conocida por su incapacidad de entablar diálogos y no poseer voluntad de abrirse a los nuevos caminos epistemológicos que ofrece la Racionalidad Ambiental.

La teoría de Leff se puede interpretar como una nueva opción de ser del Ser, una alternativa a la racionalización instrumental que agudiza la crisis ambiental, que en realidad es una crisis de la naturaleza (LEFF, 2004, p.259), causada por la externalización del ambiente del sistema económico, la tergiversación del concepto ontológico de la misma naturaleza, tomada como legitimadora del proceso de racionalización y validadora de las leyes interpuestas por la ciencia como leyes de ella misma, que además de inmutables, niegan la naturaleza dinámica de la naturaleza.

En coherencia con el argumento anterior, Enrique Leff plantea una nueva relación humano-mundo, cimentada en una nueva epistemología del ambiente, una nueva visión del desarrollo sostenible, en una nueva racionalidad, una Racionalidad Ambiental, que define como:

La racionalidad ambiental es una categoría que aborda las relaciones entre instituciones, organizaciones, prácticas y movimientos sociales, que atraviesan el campo conflictivo de lo ambiental y afectan las formas de percepción, acceso y usufructo de los recursos naturales, así como la calidad de vida y los estilos de desarrollo de las poblaciones. (LEFF, 2004, p.200).

En ese orden de ideas, la Racionalidad Ambiental se muestra como "un proceso racionalizador y transformador de formaciones ideológicas" (LEFF, 2004, p.223), alejadas de las construcciones propias de una racionalidad económica instrumental con un brazo operativo llamado globalización, e interpretado como un proceso homogeneizante que incluye la negación de las demás culturas y negación sistemática de otros estilos de desarrollo.

Es importante dar cuenta de la penetración de esas construcciones en los resquicios de nuestra sociedad. En este caso decidimos indagar la penetración de la racionalidad económica en la Educación Ambiental del municipio de Valledupar, inspeccionando los documentos correspondientes a 
los PRAE de las instituciones oficiales. Posterior a este análisis logramos establecer dos categorías de análisis guiadas por la teoría de Racionalidad Ambiental, en procura de simplificar las interpretaciones.

\section{Objetivación de la naturaleza en los PRAE}

La naturaleza objetivada por una racionalidad instrumental corresponde a los procesos de asignación de Valor a los recursos naturales y culturales por parte de la economía, recursos que pueden y deben usarse en la producción de bienes y servicios para satisfacer las necesidades anidadas en el ser actual (MARCUSE, 1969 apud SARMIENTO, 2018) consecuentemente con la posibilidad de estar insertada en los programas de educación Ambiental, específicamente en los PRAE.

Esto está ciertamente constatado en el análisis documental realizado, donde se encontró la prevalencia del tratamiento de los residuos sólidos como problemática principal en los diagnósticos de las instituciones analizadas (Figura 1). Esta problemática es reforzada en objetivos fundamentados en un pensamiento ecológico neoliberal, que piensa en la ecologización de la economía, pero no establece límites al crecimiento (LEFF, 2004, p.181); y que está enfocados a solucionar problemas estéticos de las instalaciones, en lugar de fomentar actividades que incentiven una verdadera conciencia ambiental.

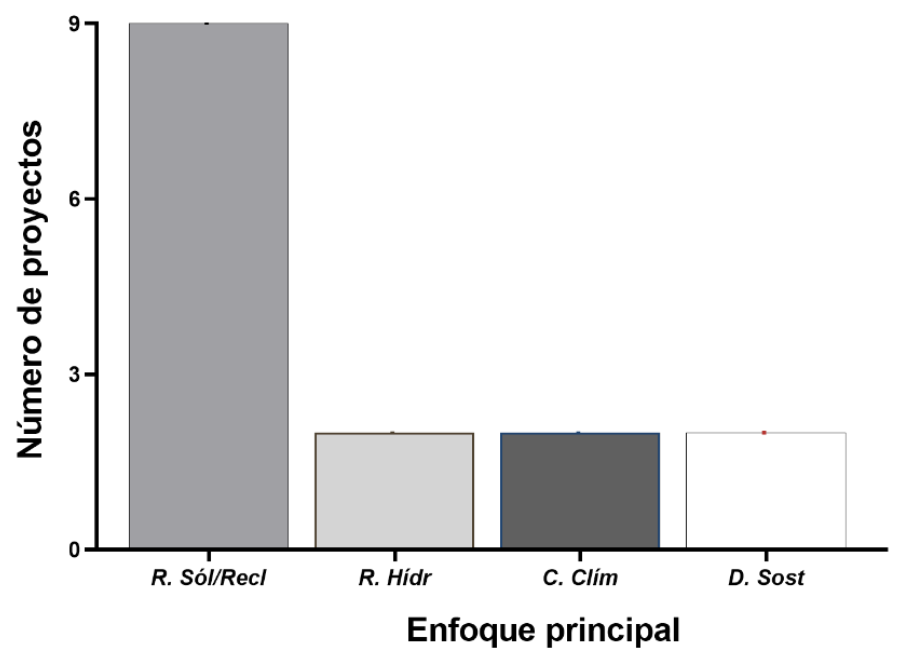

Figura 1: Enfoques en los Proyectos Ambientales Escolares. Donde: R. Sól/Recl: Residuos sólidos/Reciclaje, R. Hídr: Recursos hídricos, C. Clim: Cambio climático, $D$. Sost: Desarrollo sostenible.

Fuente: Elaborada por los autores.

El problema central que descubrimos en los PRAE es la incoherencia entre la justificación del problema, que se muestra guiada por los lineamientos del desarrollo sostenible y los valores ambientales, frente a las actividades propuestas para lograr transmitir esos conocimientos. De modo que, 9 de los 12 proyectos analizados, proponen actividades enfocadas en el reciclaje y 
gestión de los residuos, complementadas con celebraciones del calendario ambiental.

En resumen, en los documentos oficiales se plantea una Educación Ambiental para crear conciencia ecológica, e interdependencia con la naturaleza. No obstante, se presenta el reciclaje de los residuos como eje principal del problema. Prácticamente, se da por sentado que, una vez resuelta la problemática de la disposición de los residuos sólidos, se resolverán las presiones al ambiente.

Aunque la Racionalidad Ambiental se fundamenta en valores como la calidad de vida, a la cual abogan los PRAE analizados, en los objetivos de las actividades realizadas en las instalaciones, según los explica Leff (2012a, p.50), esta no puede depender de actividades que objetiven la naturaleza como son las campañas atomizadas de reciclaje en las instituciones que no tienen una disposición final adecuada, causando una abstracción de los problemas ambientales.

\section{Alienación de los participantes en los PRAE}

El término Alienación es definido por la Real Academia Española (RAE) como "Limitación o condicionamiento de la personalidad, impuestos al individuo o a la colectividad por factores externos sociales, económicos o culturales" (RAE, 2020). En este caso particular, se puede interpretar como "la subordinación de valores humanos al interés económico e instrumental" (LEFF, 2004, p.185) de los individuos que hacen parte los PRAE analizados, en los cuales se descubre una potencialidad de predisponer el público de los proyectos a prácticas de consumo descontrolado y objetivación de la naturaleza, lo que puede desencadenar en una sobreconomización de los recursos que se traduce en una barrera para lograr el desarrollo sostenible.

Los PRAE sufren el influjo de las relaciones de poder vigentes entre los territorios latinoamericanos y las potencias mundiales que tienen influencia en la creación e implementación políticas públicas. Es evidente que los estados desmejoran el ambiente con el fin de llegar a un nivel de desarrollo "ideal", correspondiente a un patrón diseñado por las mismas potencias, las cuales no tienen en cuenta los contextos locales y se autoproclaman no solo como "el orden social deseable, sino en el único posible" (LANDER, 2000, p.4).

Partiendo de los supuestos inmediatamente anteriores, es necesario repensar la Educación Ambiental en nuestros territorios y lograr desarrollar programas con narrativa propia de acuerdo con las problemáticas locales.

\section{Diálogo de saberes alternativa para la Educación Ambiental en Colombia}

La crisis ambiental puede ser interpretada de diferentes formas, sin embargo, la mayoría de las veces es interpretada de manera simplista como una crisis de los recursos naturales, la cual es una interpretación influenciada

Revbea, São Paulo, V. 16, № 2: 370-384, 2021. 
por la teoría de valor. En cambio, Enrique Leff la define como "una crisis de civilización producida por el desconocimiento del conocimiento" (LEFF, 2012a, p.57), y causada principalmente por la estigmatización de los conocimientos que no son frutos del positivismo. Esta crisis puede ser superada pensando en otras formas de saber apartadas, de cierto modo, de la creencia que privilegia al saber científico como el único conocimiento válido e incuestionable.

Carrizosa define a Colombia de forma poética como un "territorio fruto del azar geológico y climático en la esquina noroeste de América del Sur" (CARRIZOSA, 2014, p.17), un país lleno de particularidades con situaciones sociales, económicas, culturales y ambientales particulares. Desde ese punto de vista, y como cualquier otro país del mundo, Colombia se hace merecedora de soluciones particulares, entre estas, una Educación Ambiental particular. Estrategia que consideramos eje central para superar la crisis ambiental, social, económica y cultural. Una Educación Ambiental basada en las potencialidades de la sabiduría ancestral de los pueblos, reforzada por las raíces ecológicas y culturales, y desarrollada mediante un encuentro de saberes en las instituciones educativas del territorio nacional.

El diálogo de saberes nos estimula a pensar en un paradigma alejado de la dominación del saber derivado de un "discurso compuesto de representaciones, imaginarios y prácticas de corte eurocéntrico con capacidad de influenciar a las sociedades del sur desde ese eje geo-económico y político del mundo" (GONZÁLEZ, 2017, p.4); y nos incita a transitar por sendas diferenciadas hacia la sustentabilidad, a abrir la historia para apoyarnos en ella y lograr un intercambio de experiencias. En palabras de Enrique Leff:

El diálogo de saberes convoca y se inserta en una política de la interculturalidad, que se plantea en el campo estratégico del posicionamiento de actores sociales ante la reapropiación social de la naturaleza y la construcción de un futuro sustentable. (LEFF, 2004, p.336).

En resumen, el diálogo de saberes plantea un intercambio de significaciones entre las ciencias y los saberes no científicos, que podríamos denominar conocimientos locales, muchas veces ancestrales, y que aún no han querido globalizarse ni ser objetos del mercado económico.

Latinoamérica es por demasía un territorio propicio para entablar un diálogo de saberes capaces de pensar el desarrollo desde otra perspectiva. Un diálogo que propicia un consenso producto del encuentro del Yo en lo Otro, con un enfoque de Conocer desde el sur, capaz de reivindicar la cosmovisión de las sociedades latinoamericanas. Enrique Leff en su libro "Racionalidad Ambiental: la reapropiación social de la naturaleza" (2004), dedica especial atención a aquellas comunidades 0 sociedades latinoamericanas que aplican tal metodología para lograr un desarrollo, social, económico y cultural, en 
concordancia con la naturaleza. Casos puntuales en seringueiros, zapatistas, afrodescendientes y pueblos indígenas de américa latina.

Es prudente aclarar, que no estamos planteando una ruptura total con las metodologías de occidente cuando hablamos de pensar en el desarrollo desde otra perspectiva, basándonos en los conocimientos locales de los pueblos. Se trata más bien de permear esas metodologías con saberes locales sobre el uso y transformación de la tierra, alejados de la sobreconomización de la naturaleza, que es un enfoque claramente contradictorio a las pretensiones de la sustentabilidad como modelo económico y social. Lo anterior con el objetivo de conservar los recursos a los que tienen derecho las futuras generaciones.

A sabiendas de que, en la actualidad en la región, aún existen comunidades que conservan sus tradiciones de uso de la tierra armónicos con los ciclos naturales de la naturaleza, el diálogo de saberes en la Educación Ambiental colombiana se muestra como una alternativa factible y con resultados prometedores en el escenario de significaciones latinoamericanas a los conceptos de crisis ambiental, para desarrollar un "Pensamiento ambiental Latinoamericano" (LEFF, 2012b), que son necesarios para encontrar soluciones propias desde la diversidad cultural, soberanía nacional y autonomías locales, que solo puede tener éxito si está permea estadios de la sociedad como la educación.

Esto implica el desarrollo una Educación Ambiental libertaria y emancipadora, fundada bajo pilares del saber ambiental latinoamericano, arraigado en el potencial cultural y los saberes de los pueblos del Sur (LEFF, 2012 b, p. 2). Esa educación emancipatoria ya pensada por Paulo Freire, entre otros pensadores latinoamericanos, como "una educación capaz de construir una consciencia de las condiciones de opresión entre opresores y oprimidos" (SAMPAIO, 2018. p.341), necesaria para superar la colonización del saber (LANDER, 2000), y la globalización cultural de nuestros pueblos.

\section{Consideraciones finales}

Colombia es el país del realismo mágico de Gabriel García Márquez, definido por Carrizosa como un golpe de suerte de la evolución geológica del planeta (CARRIZOSA, 2014), destacado por ser un país megadiverso, no solo en diversidad biológica, sino también en diversidad cultural. En un país multicultural como Colombia no debería existir una supremacía de un solo tipo de racionalidad totalizadora con pretensiones homogeneizadoras. La multiculturalidad de un territorio debe ser traducida en las iniciativas de educación ambiental, guiadas por las cosmovisiones de cada pueblo y teniendo en cuenta el sentido propio de la naturaleza. Los PRAE son el escenario ideal para explorar esas nuevas significaciones del mundo y educar a la sociedad desde los saberes ancestrales de los pueblos nativos que viven en armonía con la naturaleza desde hace miles de años. 
Los PRAE representan una oportunidad única para Colombia como país latinoamericano repleto de encrucijadas con respecto a las decisiones que se deben tomar para alcanzar el desarrollo sostenible. Estos proyectos poseen el potencial para formar ciudadanos comprometidos con su papel de sujetos insertos en la naturaleza. Formar ciudadanos que logren pensar el ambiente no como una externalidad del mundo, sino como el todo que en realidad es, garantizará un futuro realmente sostenible. Descubrir en ellos una predominante racionalidad económica es verdaderamente preocupante. Significa que la mayoría no cumple con los objetivos básicos referentes a la formación de ciudadanos críticos frente a las problemáticas socioambientales del territorio y compromete la sustentabilidad.

Debido a que la Educación Ambiental es un pilar realmente importante en la sociedad y puede llegar a ser un aparato ideológico del estado, es imperativo hacer evaluaciones de la eficacia de las estrategias implementadas, sobre todo en la escuela por ser el espacio donde más se imparte la Educación Ambiental en la actualidad. Además, es importante insistir en la necesidad de reflexionar sobre la coherencia entre la teoría y la práctica de las políticas públicas que rigen el proceso pedagógico.

La creencia de que la Educación Ambiental consiste en una serie de actividades de celebración de un calendario de días especiales dedicados a solo algunos aspectos ambientales repercute en la calidad de la misma. Los dinamizadores se encuentran con panoramas escasos, por no decir nulo, presupuestos financiero y humano para desarrollar las actividades. Además de la sistemática desvalorización de su quehacer como actores de cambio, se suma la poca capacitación recibida por parte de los entes estatales responsables de la capacitación y actualización del personal docente y administrativo de las escuelas.

Es evidente que aún existen capítulos sin resolver en cuanto al significado de desarrollo de la humanidad. Pese a que el paradigma de "Desarrollo sin Límites", que ya se cuestionaba en la cumbre de Estocolmo en 1972, actualmente aún está vigente e insertado en el "chip" de los dirigentes y programas 0 planes de desarrollo de los países mal llamados "subdesarrollados" que comprometen sus recursos naturales y culturales a la degradación por alcanzar tal estadio de prosperidad, teñido por significaciones eurocentristas occidentalizadas, posiblemente superables con una Educación Ambiental basada en una nueva racionalidad productiva. Una racionalidad construida sobre las diferencias sobre el diálogo de saberes, una Racionalidad Ambiental.

\section{Agradecimientos}

Al Programa de Alianzas para la Educación y la Capacitación (PAEC OEA-GCUB), a la coordinación de la formación del personal de nivel superior (CAPES) y a la Universidad Federal de Alfenas, por hacer posible el desarrollo de esta investigación. 


\section{Referencias}

BUENO A. F; CERVEIRA F. J. L. Posibilidades de análisis de la acción socioambiental según la racionalidad ambiental en Enrique Leff, Tabula Rasa, n. 24, enero-junio, 2016, pp. 171-189.

BUSTAMANTE, N. DEL C.; CRUZ, M. I.; RIVERA, C. Proyectos ambientales escolares y la cultura ambiental en la comunidad estudiantil de las instituciones educativas de Sincelejo, Colombia. Revista Logos, Ciencia y Tecnología, v. 9,n.1,p.215-229, 2017.

CARRIZOSA, J. Colombia compleja. Bogotá D.C: Jardín Botánico de Bogotá José Celestino Mutis; Instituto de Investigación de Recursos Biológicos Alexander von Humboldt, 2014.2 Disponible en: $<$ http://repository.humboldt.org.co/handle/20.500.11761/32548>. Consultado en: 5 ago. 2020.

CARSON, R. Primavera Silenciosa. Segunda Edição. São Paulo, Edições Melhoramentos, 1969.

COLOMBIA. Política nacional de educación ambiental. Ministerio de Medio Ambiente y Ministerio de Educación, Bogotá D.C, 2002. Disponible en: $<$ http://cmap.upb.edu.co/rid=1195259861703 152904399 919/politica educaci on amb.pdf>. Consultado en: 27 Jul. 2020.

COLOMBIA. Constitución Política de Colombia. p. 1-170, 1991. Disponible en:<http://www.secretariasenado.gov.co/senado/basedoc/constitucion politica 1991.html>. Consultado en: 27 jul. 2020.

COLOMBIA. Ley 115 del 1994 "Ley general de educación”. Congreso de la Republica, Bogotá D.C, 1994. Disponible en: $<$ https://www.mineducacion.gov.co/1621/articles85906 archivo pdf.pdf>.Consu Itado en: 27 Jul. 2020.

COLOMBIA. Decreto no 2811, de 1 de dezembro de 1974. Bogotá: Congreso de la República, $1974 . \quad$ Disponible en: $<$ https://www.minambiente.gov.co/images/GestionIntegraldelRecursoHidrico/pdf /normativa/Decreto 2811 de 1974.pdf $>$. Consultado en: 1 ago. 2020.

COLOMBIA. Decreto ${ }^{\circ}$ 1337, de 10 de Julio de 1978. Bogotá D.C. Ministerio de Educación Nacional. Disponible $<$ https://www.minambiente.gov.co/images/BosquesBiodiversidadyServiciosEcos istemicos/pdf/Normativa/Decretos/dec 1337 100778.pdf>. Consultado en: 1 ago. 2020.

DE SOUSA SANTOS, B. Conocer desde el Sur: Para una cultura política emancipatoria. Lima: UNMSM, 2006.

ESCHENHAGEN, M. L. Las cumbres ambientales internacionales y la Educación Ambiental, Oasis, $\mathrm{n}^{\circ}$ 12. 2007.

GALANO, C. et al. Manifiesto por la vida por una ética para la sustentabilidad. Ambiente \& Sociedade, 5 (10), 1-14, 2002. 
GIL, A. C. Como elaborar projetos de pesquisa. 4. ed. São Paulo: ATLAS S.A, 2002.

GONZÁLEZ, C. H. La Educación Ambiental ante el problema ético del desarrollo. Revista Electrónica Educare, v. 21, n. 2, p. 296-314, 2017.

HERRERA, C. R. et al. Evaluación de los proyectos ambientales escolares en colegios oficiales de la localidad 18 en Bogotá. Gestión y Ambiente, v. 9, n. 1, p. 115-122, 2006.

HUÉRFANO, G. 2018. Proyectos Ambientales Escolares (PRAE) cómo estrategia de implementación de la Educación Ambiental en la educación básica y media en Colombia. Disertación (Maestría en Ciencias Ambientales y conservación) - Programa de posgraduación en ciencias ambientales y Conservación, Universidad Federal do Rio De Janeiro.

IRAZO, J.M. Meadows, Donella; Randers, Jorgen y Meadows, Dennis, Limits to Growth: The 30-Year Update. Chelsea Green, 2004. EMPIRIA. Revista de Metodología de Ciencias Sociales. N. ${ }^{\circ}$ 10, julio-diciembre, 2005, pp. 227-245.

LANDER, E. (ed.), La colonialidad del saber, Buenos Aires, CLACSO/UNESCO. 2000.

LEFF, E. Racionalidad Ambiental: la reapropiación social de la naturaleza. Primera. ed. Ciudad de México: 2004. Disponible en: $<$ http://ru.iis.sociales.unam.mx/jspui/bitstream/IIS/4937/1/Racionalidad ambient al.pdf>. Consultado en: 20 jun. 2020.

LEFF, E. Epistemologia ambiental. (2ª - ed.). SP: Cortez. 2002.

LEFF, E. Aventuras da epistemologia ambiental: da articulação das ciências ao diálogo de saberes. São Paulo: Cortez. 2012a.

LEFF, E. Pensamiento Ambiental Latinoamericano: Patrimonio de un Saber para la Sustentabilidad. Environmental Ethics, v.34, p. 97-112, 2012b..

MINISTERIO DE EDUCACIÓN NACIONAL. Decreto no 1743, de 03 de agosto de 1994. Bogotá. 1994. Disponible en:

$<$ https://www.minambiente.gov.co/images/BosquesBiodiversidadyServiciosEcos istemicos/pdf/Normativa/Decretos/dec 1743 030894.pdf>. Consultado en: 24 jul. de 2020.

ORGANIZACIÓN DE LAS NACIONES UNIDAS, informe de la conferencia de las naciones unidas sobre el medio humano, New York, 1973. Disponible en: $\quad<h$ ttps://archivos.juridicas.unam.mx/www/bjv/libros/8/3614/10.pdf>. Consultado en: 21 jul. 2020.

OTERO-COTES, A., HERRERA, F., MONROY, J, M. Análisis de la Pobreza y Condiciones de Vida en Valledupar, Banco de la Republica, Cartagena, Colombia, 2019.

PIZANO. C, GARCIA, H. (Eds). Bosque seco tropical em Colombia. Instituto de recursos biológicos Alexander Von Humboldt. Bogotá D.C, Colombia. 2014. 
REAL ACADEMIA ESPAÑOLA. Diccionario de la lengua española, 23. ed., [versión 23.3 en línea]. 2020. Disponible en: <https://dle.rae.es>. Consultado en: 30 jul. 2020.

RODRIGUEZ, M. Hacer más verde al Estado Colombiano? Revista de Estudios Sociales, v. 32, n. 32, p. 18-33, 2009.

RODRIGUEZ, M. et al. Gobernabilidad, instituciones y medio ambiente en Colombia. Foro Nacional Ambiental. Bogotá D.C: 2008.

SAMPAIO, R, O. Análise da década da educação para o desenvolvimento sustentável (DEDS) da UNESCO a partir da leitura da pedagogia da autonomia de Paulo Freire, Revista brasileira de educação ambiental, São Paulo, V. 13, No 2: 340-350, 2018.

SARMIENTO, Y. H. A. 2018. EDUCAÇÃO AMBIENTAL E O PENSAMENTO CRÍTICO: uma aproximação à Política Nacional de Educação Ambiental da Colômbia. Disertación (Maestría em Ciencias Sociales) Programa de posgraduación en ciencias sociales, Universidade Estadual Paulista. Disponible en: <https://repositorio.unesp.br/handle/11449/155867>. Consultado en: 25 jul. 2020.

SECRETARIA DE EDUCACIÓN MUNICIPAL. Reporte de matrícula oficial 2020, municipio de Valledupar, Colombia. 2020.

TORRES, M. La Educación Ambiental: una estrategia flexible, un proceso y unos propósitos en permanente construcción. La experiencia de Colombia. Revista Iberoamericana de Educación, v.16. p. 23-48. 1998. 